\title{
CARACTERIZAÇÃO DA PERFORMANCE HIDRÁULICA DE DOIS EMISSORES TIPO MICROASPERSOR ${ }^{1}$
}

\author{
José de Arimatea de Matos e Carlos Renato Alves Ragoso ${ }^{3}$
}

\begin{abstract}
RESUMO
Avaliaram-se as características hidráulicas de dois emissores de irrigação localizada, tipo micoraspersor Carborundum, modelo MSIII, tendo sido analisados o coeficiente de variação de fabricação e a equação característica da relação vazão-pressão. De acordo com a classificação da ASAE, quanto ao coeficiente de variação de fabricação os microaspersores avaliados são considerados médio. Com os dados obtidos da relação de vazão versus pressão encontrou-se a equação característica de cada microaspersor. $O$ de bocal verde claro apresentou a quação $q=6,9235 \mathrm{H}^{0,3804}$, com coeficiente de determinação $\left(\mathrm{R}^{2}\right)$ de 0,98 e regime de fluxo considerado plenamente turbulento. $\mathrm{O}$ de bocal rosa apresentou equação $q=28,78 \mathrm{H}^{(1.185 .3}$, com um $\mathrm{R}^{2}$ igual a $0,80 \mathrm{e}$, de acordo com seu expoente de descarga, é considerado auto-compensante; quanto ao fenômeno de histerese, sua amplitude foi considerada desprezível.
\end{abstract}

Palavras-chave: características hidráulicas, irrigação localizada, microaspersor

\section{HYDRAULIC PERFORMANCE CHARACTERIZATION OF TWO MICROSPRINKLER TYPE DRIPPERS}

\begin{abstract}
The hydraulic characteristics of two trickle irrigation emitters, type micro-sprinkler Carborundum, model MSIII, were evaluated by analyzing the manufacturing variation coefficient and pressure discharge relationship. According to the ASAE classification, in relation to the manufacturing variation coeflicient, the evaluated micro-sprinkler are considered as average. With the data obtained from the discharge versus pressure, the characteristic equation of each micro-sprinkler was found. The one with light green socket presented an equation $q=6.9235 \mathrm{H}^{0.3804}$, with a determination coefficient $\left(\mathrm{R}^{2}\right)$ equal to 0.98 and flow regime considered completely turbulent. The one with pink socket presented an equation $\mathrm{q}=28.78 \mathrm{H}^{0.0853}$, with $\mathrm{R}^{2}$ equal to $0.80 \mathrm{and}$, according to the exponent discharge value, it is considered as self-compensating. About the hysteresis phenomenon, its amplitude was considered negligible.
\end{abstract}

Key words: hydraulic characteristics, trickle irrigation, micro-sprinkler

\section{INTRODUÇÃO}

A prática da irrigação, quando adequadamente utilizada, torna-se um eficiente instrumento na elevação da renda do produtor, além da geração de empregos e, consequentemente, aumento da oferta de produtos agrícolas. Um bom sistema de irrigação deve aplicar água no solo uniformemente, até determinada profundidade, proporcionando umidade necessária ao desenvolvimento normal das espécies vegetais.

Dentre os métodos de irrigação, a localizada vem sendo a mais utilizada nas regiōes de maior escassez de água. O grande interesse despertado pelo sistema, mesmo onde há abundância

\footnotetext{
${ }^{1}$ Pesquisa financiada pela FAPESP, processo 97/00262-0.

${ }^{2}$ M.Sc. Irrigação e Drenagem do DEAG/UJFPB, Doutorando em Irrigação e Drenagem, FCA/UNESP, CP., 237, CEP 18.603-970, Botucatu, SP, Fone (014) 821.3883, Fax (014) 821.3438.

${ }^{3}$ Estudante de Pós-Graduação em Agronomia - Irrigação e Drenagem FCA/UNESP, CP. 237, CEP 18.603-970, Botucatu, SP, Fone (014) 821.3883, Fax (014) 821.3438.
} 
de água, deve-se ao fato de sua alta eficiência de aplicação de água e ao seu elevado potencial para automação total ou parcial, além de pequena utilização de mão-de-obra, já que possibilita a aplicação de fertilizantes e herbicidas na água de irrigação.

Os dois principais sistemas onde se emprega o método de irrigação localizada são a microaspersão e o gotejamento. A microaspersão foi idealizada para substituir o gotejamento em áreas de frutíferas localizadas em solos arenosos, onde se necessita de um bulbo molhado de maiores dimensões para que atendam às necessidades mínimas de área molhada.

Os emissores são denominados microaspersores e se constituem nos elementos de maior importância dos sistemas de irrigação por microaspersão. São definidos como sendo uma estrutura mecânica utilizada para dissipar a pressão da água nas tubulações laterais, de modo que possibilite a distribuição da vazão de forma frequiente, uniforme e constante.

O objetivo desse estudo foi avaliar as características hidráulicas, como coeficiente de variação de fabricação e equação característica de dois emissores tipo microaspesores, modelos MSIII da Carborundum, adquiridos com recursos da Fundação de Amparo à Pesquisa do Estado de São Paulo FAPESP, para o projeto de pesquisa "Eficiência de aproveilamento de nutrientes pelo cilrus aplicado via fertirnigação em comparação com a adubação convencional”.

\section{MATERIAL E MÉTODOS}

Os dois modelos de emissores foram ensaiados no Laboratório de Irrigação do Departamento de Engenharia Rural da ESALQ, em Piracicaba, ambos com vazão nominal de $43 \mathrm{l} / \mathrm{h}$ e diâmetro do orifício de saída de água de $1,03 \mathrm{~mm}$, sendo um de bocal verde claro e o outro com bocal rosa. A amostra foi constituída de 20 unidades de cada modelo, escolhidos aleatoriamente, conforme as recomendações da ASSOCIAÇÃO BRASILEIRA DE NORMAS TÉCNICAS (1986).

\section{Cocficiente de variação de fabricação}

Para se avaliar as variações de vazões que ocorrem entre os microaspersores em virtude de diferenças inerentes ao processo de fabricação, os emissores selecionados foram submetidos a uma pressão de trabalho recomendada pelo fabricante de $150 \mathrm{kPa}$, durante um intervalo de tempo de 6 minutos. Segundo Abreu et al. (1987) o coeficiente de variação de fabricação é praticamente independente da pressão usada no teste, sempre que esta esteja compreendida na faixa de funcionamento do emissor.

A água foi coletada através de medida direta, em seguida pesada em balança eletrônica e transformada em volume, que posteriormente foram convertidos em vazões $(1 / \mathrm{h})$, sendo o seu resultado a média aritmética de três repetições.

A parlir dos dados de vazão, calcularam-se a vazão média e o desvio padrão (Eq. 1); em seguida, determinou-se o Coeficiente de Variação de Fabricação (CVF), dividindo-se o desvio-padrão pela média da vazão (Eq. 2).

$$
\bar{s}=\sqrt{\frac{\sum_{i=1}^{n}\left(q_{i}-4\right)^{2}}{n-1}}
$$

sendo:

$\delta=$ desvio-padrão;

$\mathrm{q}_{\mathrm{i}}=$ vazão de cada emissor e,

$\mathrm{q}_{\mathrm{m}}=$ vazão média dos emissores testados.

$$
C V F=\frac{\delta}{q_{m}}
$$

\section{Equação característica}

Para se determinar uma equação que relacione a vazão com a pressão na entrada do microaspersor, cada modelo foi submetido às pressões de $100,150,200,250$ e $300 \mathrm{kPa}$, com as medidas de vazões realizadas da mesma forma que para o coeficiente de variação de fabricação.

Tendo por finalidade analisar o fenomeno de histerese na relação vazão-pressão, o modelo de microaspersor autocompensante foi submetido a pressões crescentes e posteriormente decrescentes.

Por recomendação do INSTITUTO ESPANHOL DE NORMALIZACION, citado por Nogueira \& Gornat (1990) os microaspersores do modelo aulo-compensante, antes de se iniciar o ensaio, foram acondicionados, sendo submetidos a pressão nominal de $200 \mathrm{kPa}$, até estabilização; em seguida, foram submetidos a pressão máxima $\left(\mathrm{P}_{\max }\right)$ e pressão mínima $\left(\mathrm{P}_{\min }\right)$, alternadamente, por três vezes. Essas pressões extremas foram mantidas, em cada operação, durante um tempo mínimo de 3 minutos.

Com as vazões médias das 20 unidades e sua respecliva pressão foi determinada a equação que relaciona a vazão com a pressão do emissor, (Eq. 3).

$$
q=K \cdot h^{x}
$$

sendo:

$\mathrm{q}=$ vazão do emissor $(1 / \mathrm{h})$

$\mathrm{K}=$ constante de proporcionalidade, que caracteriza cada emissor;

$\mathrm{h}=$ pressão hidráulica à entrada de água no emissor $(\mathrm{kPa}) \mathrm{e}$, $\mathrm{x}=$ expoente de emissão, parâmetro que caracteriza o fluxo de um emissor como uma função da pressão de operação.

\section{RESULTADOS E DISCUSSÃO}

\section{Coeficiente de variação de fabricação}

O modelo de microaspersor de bocal verde claro, apresentou, para a pressão de $150 \mathrm{kPa}$, uma vazão média de 48,41 1/h (Tabela 1 ) com desvio-padrão de 2,92 1/h e coeficiente

Tabela 1. Valores médios de vazão para cada pressão de ensaio e o valor fornecido pelo labricante para o microaspersor Caborundum MSIII de bocal verde claro.

\begin{tabular}{cccc}
\hline $\begin{array}{c}\text { Pressão } \\
(\mathrm{kPa})\end{array}$ & $\begin{array}{c}\text { Vazão ensaiada } \\
(\mathrm{l} / \mathrm{h})\end{array}$ & $\begin{array}{c}\text { Vazão do fabricante } \\
(\mathrm{l} / \mathrm{h})\end{array}$ & $\begin{array}{c}\text { Erro sistemático } \\
(\%)\end{array}$ \\
\hline 100 & 38,99 & 35,3 & 10,5 \\
150 & 48,41 & 43,0 & 12,6 \\
200 & 52,01 & 50,9 & 2,2 \\
250 & 56,01 & 57,0 & $-1,7$ \\
300 & 60,19 & 63,8 & $-5,7$ \\
\hline
\end{tabular}


de variação de fabricação de 0,069 . Para esta mesma pressão o modelo de bocal rosa forneceu uma vazão média de $42,79 \mathrm{l} / \mathrm{h}$, apresentando desvio-padrão de $2,921 / \mathrm{h}$ e coeficiente de variação de fabricação de 0,068. Com esses valores de CVF, os dois modelos de microaspersores são considerados de classificação média, segundo a ASAE (1995).

Verifica-se, nas Tabelas 1 e 2 que o valor médio da vazão foi de apenas $0,5 \%$ inferior ao valor fornecido pelo fabricante para o microaspersor de bocal rosa, enquanto que para o de bocal verde-claro a vazão média foi superior em $12,6 \%$.

Tabela 2. Valores médios de vazão (l/h) do microaspersor de bocal rosa, obtidos a partir de pressões crescentes e decrescentes

\begin{tabular}{ccccc}
\hline & \multicolumn{2}{c}{ Va/üo $($ l/h) } & \multicolumn{2}{c}{ Frro Sistemálico } \\
\cline { 2 - 5 } Pressăo $(\mathrm{kPa})$ & P. Crescente & P. Decrescente & P. Cresecente & P. Decrescente \\
\hline 100 & 43,35 & 40,13 & 0,8 & $-6,7$ \\
150 & 42,79 & 40,44 & $-0,5$ & $-6,0$ \\
200 & 45,28 & 43,78 & 5,3 & 1,8 \\
250 & 46,60 & 45,61 & 8,4 & 6,1 \\
300 & 46,86 & 46,86 & 9,0 & 9,0 \\
\hline
\end{tabular}

\section{Equação Característica}

A partir dos dados de vazão e sua respectiva pressão determinou-se a equação característica de cada modelo e confeccionou-se a curva característica. A Figura 1 mostra a curva característica do microaspersor de bocal verde-claro, cujos dados ajustados resultaram na equação $\mathrm{q}=6,9235 \mathrm{H}^{0,3804}$, com coeliciente

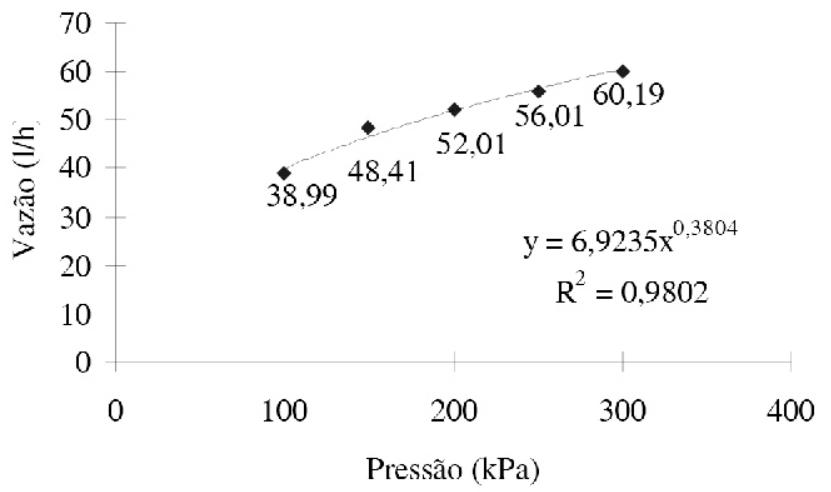

Figura 1. Curva característica do microaspersor Carborundum MSIII, de bocal verde.

de determinação $\left(\mathrm{R}^{2}\right)$ de 0,98 . Com o valor do expoente de descarga ( $x=0,38)$, Keller \& Karmeli (1974) classificam o seu fluxo como plenamente turbulento. Como os valores de vazão ensaiados divergem dos fornecidos pelo fabricante (Tabela 1) há divergência também na equação que é $\mathrm{q}=2,9316 \mathrm{H}^{0,5385}, \mathrm{com}$ $\mathrm{R}^{2}$ igual a 0,998 e fluxo classificado como parcialmente turbulento.

A Figura 2 mostra a curva característica do microaspersor de bocal rosa, que possui a equação potencial que define o emissor $\mathrm{q}=28,78 \mathrm{H}^{0,0853}$, com $\mathrm{R}^{2}$ igual a 0,80 . Como o valor do expoente de descarga está próximo de zero, este emissor é considerado auto-compensante (Keller \& Karmeli, 1974) e, sendo assim, para esse emissor, foram determinadas vazões para pressões crescentes e decrescentes, com a finalidade de observar o fenômeno de histerese. A Tabela 2 mostra estas vazões, cujas variações para pressão crescente em relação à vazão nominal fornecida pelo fabricante foi de $-0,5 \%$ a $9 \%$.

Quando a pressão variou de 100 a $300 \mathrm{kPa}$, ocorreu variação da vazão de $9 \%$ e de $15,4 \%$, respectivamente, para

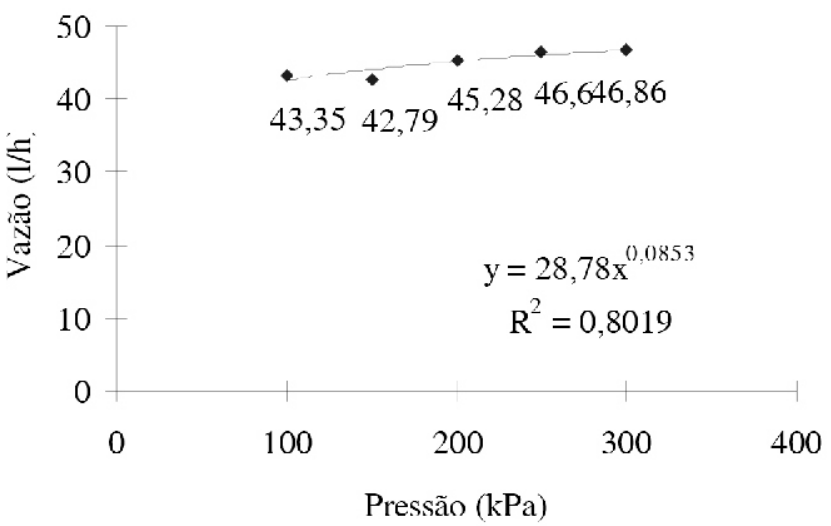

Figura 2. Curva característica do microaspersor Carborundum MSIII de bocal rosa.

pressões crescente e decrescente. Na elaboração de projetos de irrigação costuma-se usar uma variação de vazão de $10 \%$, sendo assim, a variação ocorrida na pressão crescente é satisfatória. Quanto à variação de vazão na pressão decrescente não tem grande importância, já que esta só ocorre nos momentos de desligamento do sistema.

A partir dos dados apresentados na Tabela 2, confeccionaram-se as curvas de vazão versus pressão (Fig. 3). Observa-se uma pequena variação de vazão entre as pressões crescente e decrescente, tornando o fenomeno de histerese praticamente inexistente. Matos et al. (1996) também observaram as mesmas condições, quando estudaram o microaspersor autocompensante Dan sprinkler 2001; no entanto, Nogueira e Gornat (1990), Bordignon \& Testezlaf (1993) e Lucena (1993), observaram, em gotejadores auto-compensantes, comportamento diferenciado, ou seja, grandes variações de vazão, quando o sistema começava a funcionar com uma pressão baixa e ia adquirindo pressão e vice-versa. Tal como aconteceu nesta pesquisa, os autores citados sempre obtiveram valores maiores de vazão no ensaio com pressão crescente que para pressões decrescentes, a diferença está na característica de materiais elásticos, como é o caso dos emissores auto-

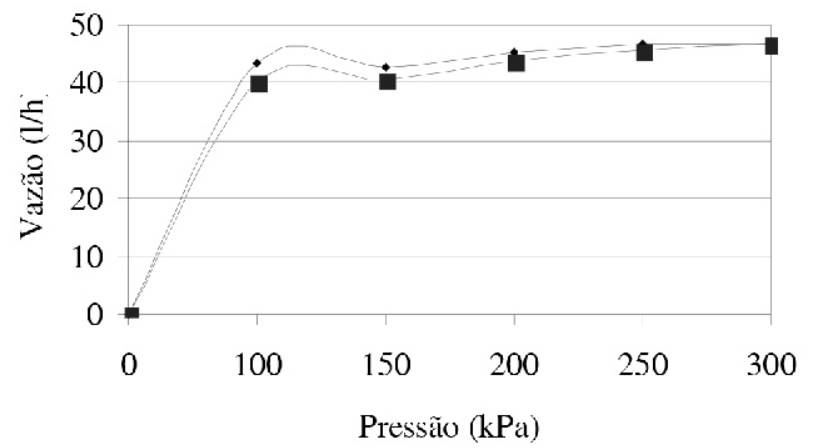

- Crescente - Decrescente

Figura 3. Fenômeno de histerese do microaspersor Carborundum MSIII de bocal rosa.

compensantes, cujo fenômeno é conhecido como histerese.

Como a abertura do diafragma do microaspersor em estudo ocorre praticamente da mesma forma para pressão 
crescente e decrescente, a amplitude do fenomeno de histerese, encontra-se nos padrões aceitáveis, de acordo com Abreu et al. (1987).

Pelos resultados mostrados nos ensaios desses microaspersores, verifica-se a importância de se analisar as características hidráulicas dos emissores, antes de se usá-los em projetos de irrigação, tendo em vista que, às vezes, os calálogos fornecidos pelos fabricantes não informam a realidade de suas características, comprometendo assim o profissional que trabalha com dimensionamento de sistemas.

\section{CONCLUSÕES}

De acordo com os resultados obtidos através dos métodos usados na avaliação da performance hidráulica dos microaspersores Carborundum, modelo MSIII, de bocais verde claro e rosa, chegou-se às seguintes conclusões:

1. O microaspersor de bocal verde claro, com CVF igual a 0,069 , é classificado como médio quanto às variações que ocorrem na vazão inerentes ao processo de fabricação; quanto ao regime de fluxo, é considerado plenamente turbulento e a vazão média para a pressão de trabalho de $150 \mathrm{kPa}$, recomendada pelo fabricante, é de 48,41 1/h, superior em $12,6 \%$ à vazão de $43 \mathrm{l} / \mathrm{h}$ fornecida no catálogo do fabricante.

2. O microaspersor rosa também é classificado como médio quanto às variações que ocorrem na vazão inerentes ao processo de fabricação, com CVF igual a 0,068; quanto ao regime de fluxo, é considerado auto-compensante; para o intervalo de 100 a $300 \mathrm{kPa}$ de pressão crescente, proporcionou vazão mínima de $42,791 / \mathrm{h}$ e máxima de $46,68 \mathrm{l} / \mathrm{h}$, cujos valores são, respectivamente, inferiores em $0,5 \%$ e superiores em $8,6 \%$ ao da vazão nominal fornecida pelo fabricante e a abertura do diafragma, que funciona como regulador de fluxo, ocorre praticamente da mesma forma para pressões crescentes e decrescentes, proporcionando um fenomeno de histerese desprezível.

\section{REFERÊNCIAS BIBLIOGRÁFICAS}

ABREU, J. M. H., LOPES, J. R., REGALADO, A. P. et al. EI ricgo localizado. Madrid: Instituto Nacional de Investigaciones Agrárias, 1987. 317p.

AMERICAN SOCIETY OF AGRICULTURAL ENGINEERS. Standars enginecring pratices data: EP405.1 design and instalation of microirrigation systems. St. Joseph, 1995. p.7203.

ASSOCIAÇÃO BRASILEIRA DE NORMAS TÉCNICAS. Emissores para sistema de irrigação localizada: avaliação de características operacionais: projeto 12:02.08.21. São Paulo, 1986. 6p.

BORDIGNON W. D., TESTEZLAF. R. Normas de ensaio para gotejadores auto-compensantes. IN: CONGRESSO BRASILEIRO DE ENGENHARIA AGRÍCOLA, 22, 1993, Ilhéus. Anais... Ilhéus: Associação Brasileira de Engenharia Agrícola, 1993. v.5, p.3071-87.

KELLER, J., KARMELI, D. Trickle irrigation design parameters. Trans. $\mathbf{\Lambda} \mathbf{S} \mathbf{\Lambda} \mathbf{E}(\boldsymbol{\Lambda} \mathbf{m}$. Soc. $\Lambda$ gric. Eng. $)$, v. 17 , n.4, p.678-84, 1974.

LUCENA, K.F.M. Performance hidráulica de microgotejadores Katif novos e usados. Campina Grande, 1993. 128p. Disserlação (Mestrado em Engenharia Agrícola - Universidade Federal da Paraíba, 1993.

MATOS, J.A., DANTAS NETO, J., AZEVEDO, H.M, AZEVEDO, C.A.V. Características hidráulicas do microaspersor Dan sprinkler 2001. Irriga, v.1, n.3, p.3044, 1996.

NOGUEIRA, L. C., GORNAT, B. Desempenho de gotejador autocompensante. ITEM, n.42, p.22-8, 1990. 